\title{
Weitergabe von Patientendaten an Versicherer im Spannungsfeld von Kostendruck und Persönlichkeitsrecht
}

\author{
K. Gehring ${ }^{a}$, R. Theiler $^{b}$, P. Breitschmid ${ }^{c}$
}

Im Dschungel gesetzgeberischer Normen und angesichts divergierender Interessen der Beteiligten stellt sich für die Ärzteschaft die Frage, was an Informationen (bezüglich Indikation, Detailgrad und Gegenstand) an die Versicherer weitergegeben werden muss und darf. Die versicherungsrechtlichen Detailbestimmungen sind im Lichte höherwertiger persönlichkeitsrechtlicher Interessen der Patienten zu betrachten und es ist entsprechend zurückhaltend $\mathrm{zu}$ verfahren - Konflikte sind nicht einfach durch Aushändigung umfassenden Materials zu Lasten des Patienten (oder auf dem Umweg über den Patienten: indem ihm das Material zur Weiterleitung übertragen wird) zu lösen, da oft der Patient zur Beurteilung der Tragweite der Informationen gar nicht in der Lage ist; die fachkundige Ärzteschaft hat die Verhältnismässigkeit der von den Versicherern verlangten Informationen zu prüfen. Im Zweifelsfall und um sich nicht dem Risiko unerlaubter Auskünfte auszusetzen, wäre Klärung in einem (schieds-)gerichtlichen Verfahren anzustreben.

\footnotetext{
a lic. iur., Rechtsanwalt, ehem. wiss. Assistent Universität Zürich

b Chefarzt Rheumatologie STZ, Präsident der Ethikkommission der Stadtspitäler Triemli und Waid

c Prof. Dr. iur., Lehrstuhl für Privatrecht Universität Zürich, Ersatzrichter am Obergericht des Kantons Zürich, Mitglied der Ethikkommission der Stadtspitäler Triemli und Waid
}

Korrespondenzen:

PD Dr. med. Robert Theiler

Stadtspital Triemli

Birmensdorferstrasse 497

CH-8063 Zürich

E-Mail:

robert.theiler@triemli.stzh.ch

Prof. Dr. Peter Breitschmid

Lehrstuhl für Privatrecht

mit Schwerpunkt ZGB

Rämistrasse 74/4

CH-8001 Zürich

E-Mail:

peter.breitschmid@rwi.unizh.ch
In ihrem Artikel haben T. Mattig und C. Lutz zu «Datenschutz und Weitergabe medizinischer Daten an die Versicherer» aus Sicht der Versicherer Stellung genommen und (durchaus nicht $\mathrm{zu}$ Unrecht) für gegenseitiges Verständnis und Vertrauen zwischen Leistungserbringern und Versicherern plädiert [1]. Indes darf im «klassischen Dreiecksverhältnis» das seinerseits vielschichtige Patienteninteresse (an modernster und individuell optimaler Behandlung, Einbezug bei Behandlungsentscheiden, informationeller Selbstbestimmung sowie tiefen Kosten) nicht ausser acht gelassen werden; insbesondere muss das Bewusstsein dafür erhalten bleiben, dass vorformulierte, pauschale Einwilligungserklärungen nicht carte blanche für jeglichen Datentransfer bedeuten.

\section{Einleitung und Fragestellung}

Öffentliche Diskussion und politischer Druck führen zu teils rigiden Versuchen, im Gesundheitswesen Kosten zu sparen (z. B. Entscheid des Bundesrates betr. Alternativmedizin). Im Bestreben, die eigenen Kosten zu senken, prüfen die Versicherer ihre Leistungspflicht vermehrt und versuchen in diesem Zusammenhang, möglichst umfassende Informationen über Gesundheitszustand, Behandlungsart aber auch die übrigen
Lebensumstände der Versicherten zu erlangen, in der Hoffnung, Hinweise zu finden, um sich der Leistungspflicht entziehen zu können. Dass dabei Tatsachen, welche zugunsten der Versicherten sprechen, weniger intensiv nachgeforscht wird, liegt auf der Hand. Um die gewünschten Angaben speditiv und möglichst vollständig zu erhalten, treten die Versicherer vermehrt an die Leistungserbringer, was insbesondere bei der Ärzteschaft Unsicherheit erzeugt, ob bzw. welche Informationen an die Versicherer weitergegeben werden dürfen oder sogar müssen.

Aus juristischer Sicht ist zu prüfen, welche Rechtsgrundlagen für das Vorgehen der Versicherer bestehen und inwieweit (qualitativ und quantitativ) die Leistungserbringer zur Weitergabe von Patienteninformationen an die Versicherer berechtigt und/oder verpflichtet sind. In Bereichen, bezüglich deren keine abschliessende Beurteilung möglich ist, sollen zumindest Anhaltspunkte zur Handhabung des Informationsaustausches gegeben werden.

\section{Grund- und Interessenlagen}

Im Themenkreis rund um Informations- und Schweigepflichten sowie die konnexen Rechte sind namentlich folgende Interessen zu berücksichtigen: Einerseits haben die Versicherer ein Interesse, möglichst detaillierte Informationen über die Versicherten zu sammeln, um deren Ansprüche genau prüfen und kontrollieren zu können. Im Bereich der sozialen Krankenversicherung sind die Versicherer zur Kontrolle des Einsatzes ihrer Mittel sogar gesetzlich verpflichtet (Wirtschaftlichkeitsgebot [2], Art. 43 Abs. 6 KVG und Art. 56 Abs.1 KVG). Konkret heisst das, dass sich eine medizinische Leistung auf dasjenige Mass zu beschränken hat, welches im Interesse des Patienten liegt und für den Behandlungszweck erforderlich ist [3]. Um diese Prüfung vornehmen zu können, sind die Sozialversicherungsträger auf Informationen betreffend Gesundheitszustand und Behandlung der Patien- 
ten angewiesen. Trotzdem müssen dem Auskunftsrecht der Versicherer enge Grenzen gesetzt werden.

Die Kontrolle der Ansprüche der Versicherten würde zumindest vordergründig in deren eigenem Interesse liegen, da die Versicherer ja im Grunde lediglich die Prämien verwalten und umverteilen (Versicherungsprinzip). Diesem Interesse steht jedoch der Anspruch der einzelnen Patienten auf Persönlichkeitswahrung und Geheimhaltung sensibler Daten gegenüber. Die entsprechenden Rechtsgrundlagen sind Art. 27 ff. ZGB, die Datenschutzgesetzgebung (Bundesgesetz über den Datenschutz, DSG; SR 235.1) und im Bereich des Sozialversicherungsrechts insbesondere Art. 33 ATSG (Bundesgesetz über den Allgemeinen Teil des Sozialversicherungsrechts, SR 830.1). Eine weitere Konkretisierung enthält Art. 321 StGB (Strafgesetzbuch, SR 311.0) bei der Regelung des Berufsgeheimnisses.

Da Patientendaten in der Regel den innersten Bereich der Persönlichkeit betreffen und somit in den Schutzbereich aller genannten Gesetzesbestimmungen fallen, dürfen sie von den Leistungserbringern nur mit Einwilligung des Geheimnisherrn (Patient) an Dritte (beispielsweise Versicherer) weitergegeben werden, es sei denn, es bestehe eine anderslautende gesetzliche Regelung (Rechtfertigungsgrund).

Zur Beantwortung der Frage nach Recht bzw. Pflicht zu (sowie Umfang der) Auskunftserteilung der Leistungserbringer (Ärzteschaft und Spitäler) an die Versicherer ist somit entscheidend, ob die Weitergabe der Patientendaten durch Einwilligung des Betroffenen bzw. einer dazu befugten Stelle oder durch gesetzliche Pflichten gerechtfertigt ist. Dabei ist immer vor Augen zu halten, dass die Leistungserbringer primär im Auftragsverhältnis zu den Patienten stehen und daher (trotz Kostendruck) deren Interessen $\mathrm{zu}$ verfolgen haben.

\section{Gesetzliche Ordnung in den verschiedenen Versicherungszweigen}

\section{Allgemeine Ordnung}

Die Problematik kann aus Sicht der Leistungserbringer grundsätzlich (wenn auch nicht unproblematisch) umgangen werden, wenn der Leistungserbringer dem Versicherer gar keine Patienteninformationen (direkt) überlässt, sondern der Informationsfluss indirekt über den Patienten läuft. Die vom Versicherer erbetenen Informationen werden dem Patienten mit der Aufforderung zugestellt, sie weiterzuleiten. So hat der Patient die (allerdings nur scheinbare)
Wahl, die Daten an den Versicherer weiterzuleiten oder unter Inkaufnahme des Risikos einer möglichen Leistungsverweigerung zurückzubehalten, und der Leistungserbringer wahrt das Arztgeheimnis. Da der Patient jedoch mangels medizinischer Kenntnisse die Brisanz und Tauglichkeit der weitergeleiteten Informationen nicht beurteilen kann, ist er dem Versicherer vollständig ausgeliefert. Im Sinne einer von den Interessen der Patienten geprägten Tätigkeit ist dieses Vorgehen daher zu vermeiden.

Weiter ist die Einholung einer Entbindungserklärung [4] (Entbindung von der ärztlichen Schweigepflicht) zu erwähnen. Der Patient kann den behandelnden Arzt für die aktuelle Behandlung von seiner Geheimhaltungspflicht befreien. Normalerweise handelt es sich dabei um eine auf einen bestimmten Arzt und Sachverhalt beschränkte Entbindung vom Arztgeheimnis [5]. Da es sich bei den zur Diskussion stehenden Daten jedoch um besonders schützenswerte Daten handelt, muss - um dem Verhältnismässigkeitsprinzip (Art. 4 Abs. 2 DSG) zu genügen die Entbindung besonders klar erfolgen [6].

Häufig, jedoch problematisch, sind vom Patienten gegenüber dem Versicherer abgegebene generelle Einwilligungserklärungen, in welchen dem Versicherer das Recht eingeräumt wird, direkt beim Leistungserbringer Auskünfte zu erfragen. Werden solche beim Versicherungsvertragsabschluss abgegeben, sind sie aufgrund des Verbots übermässiger Bindung (Art. 27 Abs. 2 ZGB) als unzulässig zu betrachten [7].

Weniger problematisch mögen Entbindungserklärungen im Rahmen der Aufnahmeformalitäten bei Eintritt ins Spital erscheinen. In solchen Fällen wirkt die Entbindung in zeitlicher, persönlicher und sachlicher Hinsicht nur beschränkt. Ob allerdings die Tragweite einer solchen, in Formularen mit manch anderer Rubrik enthaltenen Erklärung für alle Patienten wirklich überblickbar sei, lässt sich kaum allgemeingültig beantworten, weshalb auch in diesen Fällen das Risiko besteht, dass eine übermässige Bindung angenommen würde [8]. Dies um so mehr, als zusätzlich danach zu differenzieren ist, was die konkret den Versicherern zufliessenden Informationen sind: ob es sich um Routinebehandlungen (Blinddarm oder Sturzverletzung) oder ein weitläufiges Prozedere mir sozialpsychischen Indikationen handelt, welches wesentlich weitreichendere Rückschlüsse zulässt. Das lässt sich jedoch häufig bei Spitaleintritt nicht absehen und die Einwilligung wäre dann immer persönlichkeitsrechtlich unzulässig. Gleiches gilt auch für entsprechende Erklärungen gegenüber freiberuflichen Ärzten. 
Nach Lehre und Praxis kann die Einwilligung für die Bekanntgabe von Patientendaten grundsätzlich auch stillschweigend bzw. konkludent erteilt werden [9]. Es stellt sich die Frage, ob die Einwilligung in die Behandlung durch den Arzt auch eine Entbindungserklärung beinhaltet. Dies ist jedoch aufgrund der hohen Sensibilität der zur Diskussion stehenden Daten grundsätzlich zu verneinen.

\section{Sozialversicherungen}

\section{Soziale Krankenversicherung nach KVG}

Im Bereich des KVG ist der Informationsfluss zwischen Leistungserbringern und Versicherern in mehreren Bestimmungen geregelt. So muss der Leistungserbringer dem Schuldner (bei «tiers payant» nach Art. 42 Abs. 2 KVG der Versicherer, bei «tiers garant» nach Art. 42 Abs. 1 KVG der Patient) eine detaillierte und verständliche Rechnung mit allen Angaben stellen, die diesem eine Überprüfung des Honorars sowie der Wirtschaftlichkeit der Leistung ermöglichen (Art. 42 Abs. 3 KVG). Zudem kann der Versicherer im Einzelfall die genaue Diagnose oder zusätzliche Auskünfte medizinischer Natur verlangen (Art. 42 Abs. 4 KVG). Verweigert der Leistungserbringer diese Auskünfte, macht er sich nach Art. 93 lit. a KVG strafbar (siehe «Schlussfolgerung»).

1. Die Unterscheidung zwischen der genügenden Rechnungsstellung (Art. 42 Abs. 3 KVG) und der detaillierteren Information (Art. 42 Abs. 4 KVG) in der Praxis ist unklar [10]. Werden wirtschaftliche Interessen denjenigen des Persönlichkeits- und Datenschutzes übergeordnet, so scheint die Forderung der Krankenversicherer nach institutionalisierter umfassender Information über den Patienten berechtigt. Dabei wird aber die Gefahr für Persönlichkeitsrechte und allenfalls berechtigte wirtschaftliche Interessen der Patienten (z.B. Problematik von verschiedenen Versicherungsprodukten nach KVG oder VVG innerhalb eines Versicherungsunternehmers) zuwenig berücksichtigt. Zudem würde bei dieser Auslegung - unter dem Deckmantel der Wirtschaftlichkeitsprüfung - die Entscheidungszuständigkeit über anzuwendende Behandlungen zunehmend vom Arzt auf den viel weniger fachkundigen und seinerseits nicht neutralen Versicherer übertragen (Fallsachbearbeiter [11] entscheiden aufgrund der ärztlichen Berichte über «wirtschaftliche Zulässigkeit» und Zweckmässigkeit einer medizinischen Behandlung), was zudem zu einer nicht erstrebenswerten Spaltung von Entscheidungsträger und allfällig Haftendem führen würde. Eine solche Entwicklung ist unsinnig und unter allen Umständen zu verhindern [12]. Neben dem Risiko von Überbehandlung durch Leistungserbringer steht ein solches der Unterversorgung sozial oder wirtschaftlich schwacher (z. B. älterer) Patienten.

In Anbetracht der geschilderten Interessen und Gefahren ist die bis anhin verbreitete Praxis, dass die Rechnung eine grobe Diagnose enthält und dem Versicherer bei Unklarheiten oder zusätzlichem Informationsbedarf in einer zweiten Phase auf Anfrage hin weitere Patientendaten bekanntgegeben werden, durchaus sachgerecht [13]. Trotzdem muss die Frage aufgeworfen werden, ob anhand der wenigen Informationen überhaupt eine Prüfung der Notwendigkeit einer Behandlung vorgenommen werden kann. Dies ist zu verneinen. Die Versicherer können anhand der Rechnung lediglich überprüfen, ob nach dem richtigen Tarif abgerechnet wurde.

Um die Aushöhlung des Persönlichkeitsschutzes (Stichwort «gläserner Patient») und die Verschiebung der medizinischen Entscheidungskompetenz weg vom Arzt, hin zur Versicherung zu vermeiden, wäre noch ein Schritt weiterzugehen und Art. 42 Abs. 3 KVG stärker im Sinne der Persönlichkeitsschutzinteressen der Patienten auszulegen. Dem Versicherer wäre das Auskunftsrecht lediglich zuzugestehen, wenn er eine Verletzung oder zumindest Indizien für die Verletzung des Wirtschaftlichkeitsgebots glaubhaft machen kann (z. B. eine deutliche Abweichung gegenüber durchschnittlichen Kosten der entsprechenden Behandlung) [14]. Damit könnte einer Tendenz zur Informationsanhäufung bei den Versicherern entgegengewirkt werden, ohne dass die in Art. 43 Abs. 6 KVG vorgeschriebene Wirtschaftlichkeitskontrolle verunmöglicht würde.

2. Nach Art. 59 Abs. 2 KVV kann zudem der Informationsfluss zwischen Leistungserbringer und Versicherer in Tarifverträgen normiert werden. Bezüglich im Tarifvertrag geregelter Leistungen sind somit von den obigen Ausführungen abweichende Regelungen möglich. Es kann also in einem Tarifvertrag vorgesehen werden, dass bereits die Rechnung eine genaue Diagnose enthalten muss [15]. Auch der TARMED enthält Bestimmungen betreffend Bekanntgabe der Diagnose bei Rechnungsstellung. Nach Art. 11 Abs. 8 lit. g TARMED-Rahmenvertrag muss bereits bei Rechnungsstellung die Diagnose nach dem vereinbarten Diagnosecode bekanntgegeben werden. Es handelt sich dabei jedoch um eine relativ grobe Einteilung, die für die Persönlichkeitsrechte der Patienten einigermassen unproblematisch ist [16]. In bezug auf künftige 
Entwicklungen ist wohl mit Forderungen nach «Verfeinerung» des Diagnosecodes zu rechnen. Eine Verfeinerung wäre jedoch mit den geltenden gesetzlichen Grundlagen nicht vereinbar und auch aus Persönlichkeitsschutzüberlegungen zu verhindern, da lediglich durch eine detailliertere Diagnoseangabe keine verbesserte Prüfung der Wirtschaftlichkeit zu erwarten ist.

Die Tarifverträge (oder aber Gesetzes- bzw. Verordnungsbestimmungen) können zusätzlich zur «nachträglichen Wirtschaftlichkeitskontrolle» im Sinne der Überprüfung der Rechnungen auch die Vornahme oder Fortsetzung einer Behandlung von der Genehmigung des Versicherers abhängig machen (Kostengutsprache). Um ein Begehren um Kostengutsprache seriös bearbeiten zu können, sind die Versicherer nicht auf die Kenntnis der Diagnose angewiesen, vielmehr sind Informationen betreffend Indikationen und Befunde notwendig, um die Wirtschaftlichkeit einer Behandlung beurteilen zu können. Ganz allgemein ist jedoch auch in den Fällen der «vorgängigen Wirtschaftlichkeitskontrolle» unbedingt zu verhindern, dass die Entscheidungskompetenz vom behandelnden Arzt auf den Versicherer übertragen wird.

3. Weiter sieht das KVG in Art. 57 die Möglichkeit der «Zwischenschaltung» eines Vertrauensarztes vor. Dieser hat die Versicherer in medizinischen Fachfragen sowie in Fragen betreffend Vergütungen und Tarifanwendungen zu beraten. Will ein Patient den in den genannten Konstellationen an sich zulässigen direkten Informationsfluss vom Leistungserbringer zum Krankenversicherer unterbinden, kann er eine entsprechende Erklärung abgeben. Hat der Patient eine solche Erklärung abgegeben, dürfen die medizinischen Angaben nicht direkt dem Krankenversicherer, sondern nur dessen Vertrauensarzt bekanntgegeben werden (Art. 42 Abs. 5 i.V.m. Art. 57 KVG). Unabhängig vom Willen des Patienten ist der Leistungserbringer in begründeten Fällen berechtigt, die vom Versicherer verlangten Angaben nur dem Vertrauensarzt bekanntzugeben (Art. 42 Abs. 5 KVG).

Durch den Beizug des Vertrauensarztes soll den Patienteninteressen Rechnung getragen werden, da dieser wie der behandelnde Arzt der Schweigepflicht untersteht und somit die sensiblen Daten nur in geringerem Umfang in die Hände der Versicherer gelangen. Dabei ist jedoch nicht zu vergessen, dass der Vertrauensarzt i.d. R. dem Versicherer sehr nahesteht und daher im Zweifellsfall zu dessen Gunsten entscheidet. Zudem ist der Informationsfluss über den Vertrauensarzt mit zusätzlichem Aufwand verbun- den, was oftmals auch nicht im Interesse der Patienten liegt [17].

\section{Soziale Unfallversicherung nach UVG}

In der sozialen Unfallversicherung statuieren Art. 45ff UVG und Art. 55 UVV die Pflicht der Versicherten und deren Hinterlassenen, alle zur Klärung des Unfallhergangs und Festsetzung der Versicherungsleistungen nötigen Daten zur Verfügung zu stellen. Werden die Informations- und Meldepflichten verletzt, trägt der Versicherte die Gefahr einer allfälligen Kürzung der Geldleistungen (Art. 15ff UVG). Zusätzlich werden die Leistungserbringer (Medizinalpersonen und Heilanstalten) in Art. 54a UVG im Bereich der Pflegeleistungen und Kostenvergütungen (Art. 10ff UVG) zu detaillierter und vollständiger Rechnungsstellung verpflichtet. Die Rechnung muss alle zur Beurteilung der Leistungsansprüche, der Berechnung der Vergütung und der Prüfung der Wirtschaftlichkeit nötigen Angaben enthalten. Bezüglich des Umfangs und Detaillierungsgrades der Informationen ist jedoch das Verhältnismässigkeitsprinzip zu beachten (siehe «Umfang der Auskunft»).

\section{Private Kranken- und Unfallversicherung nach VVG}

Im Bereich der privaten Kranken- und Unfallversicherung kommen VVG, OR/ZGB und im Bereich des Datenschutzes insbesondere das DSG zur Anwendung. Besondere Auskunftsrechte der Versicherer oder Auskunftspflichten der Leistungserbringer bestehen nicht, können jedoch im Versicherungsvertrag vereinbart werden. Patientendaten dürfen daher im Bereich der privaten Kranken- und Unfallversicherung nur mit Einwilligung der Patienten vom Leistungserbringer an den Versicherer weitergegeben werden [18]. Dabei sind die oben erläuterten Grundsätze zu beachten - namentlich kann nicht von der Zulässigkeit einer pauschalen Einwilligung ausgegangen werden, und es könnte sich im Einzelfall auch die Frage stellen, ob der vertraglich vereinbarte Umfang des Datentransfers vor den Regeln zum Schutz der Persönlichkeit der Patienten (Art. 27 Abs. 2 ZGB) standhält.

\section{Zwischenergebnis}

Im Sinne einer Zwischenbilanz ist festzuhalten, dass grundsätzlich für die Weitergabe von $\mathrm{Pa}$ tientendaten eine Einwilligung des betroffenen Patienten notwendig ist, in einzelnen Sozialversicherungszweigen jedoch aufgrund der aktuellen Gesetzeslage die Versicherer Anspruch auf Informationen betreffend in Rechnung gestellten Pflegeleistungen haben. 
Im Bereich der privaten Versicherungen (VVG) besteht keine gesetzliche Auskunftspflicht.

\section{Umfang der Auskunft}

Nachdem dargelegt worden ist, unter welchen Voraussetzungen die Leistungserbringer zur Auskunftserteilung an die Versicherer verpflichtet sind, stellt sich die wichtigere Frage nach dem Umfang der Auskunftspflicht. Zur Beurteilung von Umfang und Detaillierungsgrad, in welchem die Leistungserbringer den Versicherern Auskünfte erteilen müssen oder dürfen, ist (sowohl im Bereich der Sozial- als auch der Privatversicherungen) das Verhältnismässigkeitsprinzip massgeblich (Art. 28 Abs. 1 und 2 ATSG, Art. 4 Abs. 2 DSG) [19]. So dürfen, soweit der Patient nicht ausdrücklich und zulässigerweise (vgl. oben) weitergehende Auskünfte bewilligt hat, nur diejenigen medizinischen Auskünfte erteilt werden, welche für den Versicherer zur Prüfung der Ansprüche (Taggelder, Pflegeleistungen, Renten usw.) des Versicherten notwendig sind [20]. Dabei ist vom Leistungserbringer zu beurteilen, ob die vom Versicherer verlangten Auskünfte geeignet und erforderlich sind, um die Leistungsprüfung vornehmen zu können und ob durch die Auskunftserteilung keine höherwertigen Interessen der Patienten verletzt werden [21]. Das bedingt jedoch, dass die Versicherer entweder sehr konkrete Fragen formulieren, oder in ihren Anfragen den Leistungserbringern zumindest mitteilen, welche Leistungen geprüft werden.

Bei der Eignungsprüfung ist zu beurteilen, ob eine Auskunft an sich überhaupt der Beurteilung der in Frage stehenden Leistungen dienen kann, während bei der Erforderlichkeitsprüfung zu beurteilen ist, ob die Leistungsprüfung nicht auch durch weniger stark in die Rechte der Patienten eindringende Auskünfte möglich ist. Die Prüfung der Verhältnismässigkeit ist grundsätzlich in jedem Fall vorzunehmen, zumindest wenn keine Tarifverträge bestehen, welche den Informationsfluss zwischen Leistungserbringer und Versicherer regeln (wobei der zulässige Rahmen solcher Tarifverträge natürlich wiederum die Persönlichkeitsrechte der Patienten zu gewährleisten hätte - es können die Persönlichkeitsrechte als höhere Rechtsgüter nicht durch Tarifverträge ausgehebelt werden).

Ganz wesentlich für die Prüfung der Verhältnismässigkeit ist also die zu beurteilende Fragestellung. So können beispielsweise Informationen über frühere Krankheiten bei der Prüfung der Notwendigkeit einer Behandlung durchaus von Bedeutung sein, beim gleichen Patienten jedoch zur Beurteilung der Kausalität zwischen Unfallereignis und Gesundheitsschaden vollständig irrelevant sein. Diagnosen und Berichte, welche nicht im Zusammenhang mit den Leistungsvoraussetzungen stehen, erfüllen die genannten Voraussetzungen der Verhältnismässigkeit keinesfalls und sind weder von einer Einwilligung des Patienten noch den gesetzlichen Rechtfertigungsgründen gedeckt. Inwieweit frühere bzw. andere medizinische oder sonstige Sachverhalte (z. B. soziale) mit der aktuellen Behandlung zusammenhängen, ist im konkreten Einzelfall sorgfältig zu beurteilen. Da zur Beantwortung dieser Problematik die medizinische Beurteilung des Einzelfalls im Vordergrund steht, kann an dieser Stelle nicht weiter darauf eingegangen werden, doch impliziert diese Feststellung einen zurückhaltenden, reflektierten Umgang mit dem bestehenden Datenmaterial, das keinesfalls ungeprüft als vollständiges Dossier weitergeleitet werden darf. Um so mehr, als von den Versicherern einmal erlangte Informationen auch in einer späteren Leistungsprüfung (vor einem anderen medizinischen Hintergrund) oftmals verwendet werden, ohne die Verhältnismässigkeitsprüfung vorzunehmen.

Unter Anwendung der dargelegten Grundsätze ist auch die Bekanntgabe von Ein- und Austritts- sowie Operationsberichten zu beurteilen. Zweck dieser Berichte ist primär die Information des nachbehandelnden oder einweisenden Arztes [22]. Sie werden nicht als Grundlage zur Leistungsbeurteilung und Rechnungsstellung erstellt. Da diese Berichte oftmals Informationen enthalten, welche zur Beurteilung der Wirtschaftlichkeit der aktuellen Behandlung oder der Leistungspflicht des Versicherers weder geeignet noch notwendig sind, dürfen sie nur nach entsprechender Prüfung und gegebenenfalls mit Abdeckungen an die Versicherer weitergegeben werden [23, 24]. In diesem Sinn besteht also kein Anspruch der Versicherer auf Bekanntgabe dieser Berichte und schon gar kein Anspruch auf Leistungsverweigerung, soweit der Auskunftspflicht des Leistungserbringers anderweitig genügt worden ist.

Gleiches gilt für die Leistungserbringer, wenn der Patient eine Erklärung im Sinne von Art. 57 KVG (Vertrauensarzt) abgegeben hat. Art. 57 Abs. 6 KVG sieht vor, dass die Leistungserbringer dem Vertrauensarzt diejenigen Informationen zu liefern haben, welche er benötigt, um die Leistungspflicht des Versicherers zu beurteilen. Nach dem Wortlaut der Gesetzesbestimmung haben die Leistungserbringer also auch bei der Information des Vertrauensarztes die Verhältnismässigkeitsprüfung vorzunehmen. Dieser ist seinerseits 
ebenfalls an das Berufsgeheimnis gebunden und darf auch nur die für den Krankenversicherer zur Beurteilung der Leistung nötigen Daten weitergeben (Art. 57 Abs. 7 KVG). Allein schon aufgrund der (Vertrauens-)Stellung liegt es auf der Hand, dass Vertrauensärzte die Verhältnismässigkeitsprüfung eher zugunsten der «vertrauten» Versicherung vornehmen. Im Sinne der Interessenwahrung zugunsten der eigenen Patienten ist deshalb den behandelnden Ärzten zu empfehlen, bei der Auskunftserteilung keine Unterscheidung zwischen Versicherer und Vertrauensarzt zu machen.

\section{Mögliche Konsequenzen unerlaubter Auskunftserteilung oder -verweigerung}

Soweit ersichtlich liegt über die behandelten Fragen bislang keine, jedenfalls keine publizierte höchstrichterliche Praxis vor, weshalb die gemachten Aussagen ihre Grundlage in Gesetzestext und Lehrmeinungen haben. Die bestehenden Grauzonen erfordern kontinuierliche Klärung im Alltag, was für alle Beteiligten die Chance bietet, kontroverse Situationen zu diskutieren und gegebenenfalls (schieds-)gerichtlich überprüfen zu lassen (dazu lit. a), statt das Risiko unerlaubter Auskunftserteilung (lit. b) einzugehen:

a) Können Leistungserbringer und Versicherer bezüglich des Informationsaustausches keine Einigung finden, ist der Rechtsweg zu beschreiten. Nach Art. 57 ATSG haben die Kantone ein Versicherungsgericht als einzige Instanz zur Beurteilung der Beschwerden aus dem Bereich der Sozialversicherung einzurichten. Verschiedene Einzelgesetze sehen jedoch für Streitigkeiten zwischen Versicherern und Leistungserbringern (insbesondere Medizinalpersonen, Laboratorien oder Heilund Kuranstalten) die Zuständigkeit eines kantonalen Schiedsgerichts vor und stellen zugleich Mindestanforderungen an dessen Verfahren auf (Art. 57 UVG, Art. 89 KVG). Im übrigen richtet sich das Verfahren nach kantonalem Recht, insbesondere kommt das ATSG (Bundesgesetz über den Allgemeinen Teil des Sozialversicherungsrecht vom 6. Oktober 2000, SR 830.1) nicht zur Anwendung (Art. 1 Abs. 2 lit. a UVG, Art. 1 Abs. 2 lit. KVG) [25]. Gegen die Entscheide der Schiedsgerichte kann Verwaltungsgerichtsbeschwerde ans eidgenössische Versicherungsgericht erhoben werden (Art. 110 UVG, Art. 91 KVG).
Es geht mithin nicht an, dass in Zweifels- und Konfliktfällen Daten letztlich unbesehen weitergeleitet werden, sondern es ist ein allfälliger Konflikt vor neutraler Stelle auszutragen.

b) Als unerlaubte Auskunftserteilung kommen zwei Tatbestände in Frage. So kann ein Leistungserbringer ohne jeden Rechtfertigungsgrund Patienteninformationen dem Versicherer bekanntgegeben oder den Umfang der zulässigen Information überschritten haben. Beide Konstellationen ziehen die gleichen Rechtsfolgen nach sich. Als solche kommen einerseits Schadenersatzansprüche in Betracht, welche bei öffentlichrechtlichen Leistungserbringern nach den Regeln der Staatshaftung zu beurteilen sind und bei privaten Ärzten wohl meist von der Berufshaftpflichtversicherung übernommen werden [26]. Andererseits macht sich der unerlaubterweise Auskunft erteilende Arzt strafbar. Sofern ein Patient einen Strafantrag stellt, kann das Gericht bei vorsätzlicher Begehung eine Busse oder Gefängnis (max. 3 Jahre) aussprechen (Art. 321 Abs. 1 StGB i.V.m. Art. 36 StGB). Im Bereich der Krankenversicherung sieht Art. 93 KVG zudem für die Verletzung der Aufklärungspflicht nach Art. 42 Abs. 4 KVG die Möglichkeit der Bestrafung mit Busse oder Haft vor.

\section{Schlussfolgerung}

Im Sinne einer Schlussfolgerung aus der Auslegeordnung von Interessen, Gesetzesbestimmungen und allgemeinen Handlungsgrundsätzen ist festzuhalten, dass im Bereich des VVG allfälligen Informationsanfragen - ohne entsprechende Entbindungserklärung - keine Folge geleistet werden darf.

Im Bereich von KVG und UVG besteht grundsätzlich gegenüber dem Versicherer die Pflicht, die für die Prüfung der Leistungspflicht und der Wirtschaftlichkeit der Behandlung nötigen Auskünfte zu erteilen.

Welche Auskünfte der Versicherer benötigt, ist in allen Zweigen nach dem Verhältnismässigkeitsprinzip zu beurteilen. Zudem sind die Tarifverträge zu konsultieren. Divergenzen sind nicht einfach zu Lasten der Patienten durch Aushändigung von umfassendem Datenmaterial auszuräumen, sondern nötigenfalls in den dafür vorgesehenen Verfahren zu überwinden. 


\section{Literatu}

1 Mattig T, Lutz C. Datenschutz und Weitergabe medizinischer Daten an die Versicherer. Schweiz Ärztezeitung 2004;85(7):341-2.

2 Eugster G. Wirtschaftlichkeitskontrolle ambulanter ärztlicher Leistungen mit statistischen Methoden. Bern/Stuttgart/Wien: Haupt; 2003.

3 Kieser U. Leistungserbringer in der Krankenversicherung, Rahmenbedingungen der Tätigkeit von Leistungserbringern zu Lasten der obligatorischen Krankenversicherung. SJZ 2003;99:582.

4 Art. 321 Abs. 2 StGB, Art. 28 Abs. 2 ZGB.

5 Müller RA. Ausgewählte Gebiete der betrieblichen Kommunikation eines Spitals und Rechte der Beteiligten. Zürich: Schulthess; 2003.

6 Brühwiler-Fresey L. Medizinischer Behandlungsvertrag und Datenrecht. Zürich: Schulthess; 1996.

7 Müller [5]; vgl. BGE 108 II 59 ff. E. 3, BGE 114 Ia 350 ff. E. 6 (gegen Blankoeinwilligung in Operationen bzw. zur Bedeutung der persönlichen Freiheit im Verhältnis Arzt-Patient).

8 So Müller [5] (mit Hinweisen auf Unkenntnis eines Durchschnittspatienten bezüglich Ablauf der Zusammenarbeit Spital/Leistungserbringer und Konsequenzen der Auskunftserteilung). Man beachte die überaus strenge Praxis des Bundesgerichts bez. der Hervorhebung von Gerichtsstandsklauseln oder anderen überraschenden allgemeinen Geschäftsbedingungen in Verbraucherverträgen (für den einzelnen in der Regel weit weniger einschneidend), dazu Gauch/Schluep/ Schmid/Rey. Schweizer Obligationenrecht. Allgemeiner Teil. 8. Auflage, Zürich: Schulthess; 2003, Bd. I Rz 1140 ff., sowie zuletzt BGE 122 V 142 E. 4.c.

9 Riemer HM. Personenrecht des ZGB. 2. Auflage. Bern: Stämpfli; 2002, Rz. 371ff.

10 Eugster G, Luginbühl R. Datenschutz in der obligatorischen Krankenversicherung. In: Hürlimann B, Jacobs R, Poledna T (Hrsg.). Forum Gesundheitsrecht, Datenschutz im Gesundheitswesen. Zürich: Schulthess; 2001, S. 73-146; Häner I. Datenschutz in der Krankenversicherung, Wahrung des Patientengeheimnisses und Offenlegung von Gesundheitsdaten: eine Interessenabwägung aus Sicht des Versicherten. DIGMA 2003; Schulthess: 147.

11 Ihrerseits nach Leistungsprinzip honoriert und damit nicht notwendig neutraler.

12 Obwohl vom Gesetzgeber an sich der Weg in diese Richtung zumindest teilweise (für besonders kostspielige Untersuchungen und Behandlungen: Art. 58 Abs. 3 KVG) geebnet wurde; dazu PfiffnerRauber B. Das Recht auf Krankheitsbehandlung und Pflege. Zürich: Schulthess; 2003 (die Idee kann allerdings wohl kaum sein, dass der [unzu- länglich versorgte] Patient dann sowohl den behandelnden und den Vertrauensarzt ins Recht zu fassen hätte).

13 Bundesamt für Sozialversicherung. Beiträge zur sozialen Sicherheit, Persönlichkeitsschutz in der privaten und sozialen Kranken- und Unfallversicherung. Nr. 7/01. Bern: BSV; 2001; zur Problematik der Einführung eines standardisierten Diagnosecodes Müller [5]; Häner [10]. - Im Detail bliebe allerdings ernsthaft zu prüfen, ob diese Diagnosecodes Teil des Rechnungsinhalts sein dürfen, oder nicht separat so zu verwalten wären, dass (berechtigter) Zugriff auf diese Daten zwar möglich, aber stets nachvollziehbar ist.

14 «Gewohnheitsrechtlich» bzw. über die bekannten SIA-Normen hat sich im Kontext von Art. 375 OR (Werkvertrag) eine solche «Toleranzgrenze» von $10 \%$ entwickelt, die zwar nicht starr und schematisch anzuwenden ist (BGE 115 II 460 E. 3.b), sich aber eingelebt und bewährt hat.

15 Eugster/Luginbühl [10].

16 Selbstverständlich ist bei richtiger Betrachtungsweise nach der Persönlichkeitssensitivität unterschiedlicher Krankheitsbilder zu unterscheiden; bei kleineren ländlichen Kassen kann zudem die fehlende Anonymität ein zusätzliches persönlichkeitsrechtliches Geheimhaltungsinteresse begründen.

17 Seiler B. Konkrete Umsetzung des Datenschutzes in der Krankenversicherung. In: Hürlimann B, Jacobs R, Poledna T (Hrsg.). Forum Gesundheitsrecht, Datenschutz im Gesundheitswesen. Zürich: Schulthess; 2001, S. 168.

18 Bundesamt für Sozialversicherung [13].

19 Häner [10].

20 Müller [5]

21 Häner [10].

22 Guntern O. Datenschutz und Gesundheitswesen. In: Hürlimann B, Jacobs R, Poledna T (Hrsg.). Forum Gesundheitsrecht, Datenschutz im Gesundheitswesen. Zürich: Schulthess; 2001, S. 14.

23 Häner [10]; Merkblatt über Austritts- und Operationsberichte des schweizerischen Datenschutzbeauftragten. www.edsb.ch.

24 Bundesamt für Sozialversicherung [13].

25 Kieser U. Kommentar zum Bundesgesetz über den Allgemeinen Teil des Sozialversicherungsrechts vom 6. Oktober 2000. Zürich: Schulthess; 2003. Art. 57 N 7.

26 Häfelin U, Müller G. Grundriss des Allgemeinen Verwaltungsrechts. 4. Auflage. Zürich: Schulthess; 2002 . 